\title{
Self-control by pigeons in the prisoner's dilemma
}

\author{
FOREST BAKER and HOWARD RACHLIN \\ State University of New York, Stony Brook, New York
}

\begin{abstract}
Pigeons played a repeated prisoner's dilemma game against a computer that reflected their choices: If a pigeon cooperated on trial $n$, the computer cooperated on trial $n+1$; if the pigeon defected on trial $n$, the computer defected on trial $n+1$. Cooperation thus maximized reinforcement in the long term, but defection was worth more on the current trial. Under these circumstances, pigeons normally defect. However, when a signal correlated with the pigeon's previous choice immediately followed each current trial choice, some pigeons learned to cooperate. Furthermore, cooperation was higher when trials were close together in time than when they were separated by long intertrial intervals.
\end{abstract}

During a two-player prisoner's dilemma game, each player receives one of four possible rewards by choosing cooperation or defection, as is presented, for example, in Figure 1. The numbers in the matrix represent reward magnitude. If Player B cooperates (top row), then, in the example in Figure 1, Player A obtains 5 reward units by cooperating or 6 units by defecting. If Player B defects (bottom row), Player A obtains 1 unit by cooperating and 2 units by defecting. Player A's cooperations and defections affect Player B's rewards in the same manner.

Two aspects of the game create the dilemma. First, on any given trial, each player obtains a greater reward by defecting than by cooperating (6 vs. 5 or 2 vs. 1). Defection, therefore, maximizes reinforcement in the short term (on each trial). Second, each player obtains a greater reward if the other player cooperates ( 5 or 6 ) than if the other player defects ( 1 or 2 ). Thus, each player benefits maximally by defecting while the other player cooperates and benefits minimally by cooperating while the other player defects. As a group, the players benefit maximally if they both cooperate and minimally if they both defect. Thus, the interests of each individual player conflict with those of the players as a group.

When the game is played repeatedly, probability of reciprocation determines whether defection or cooperation will maximize reinforcement in the long term (Baker \& Rachlin, 2001). For example, imagine that Player B always reciprocates Player A's prior choice (this strategy, a 1.0 probability of reciprocation, is called tit-for-tat; Axelrod,

The research was supported by grants to the second author from the National Institute of Mental Health and the National Institute for Drug Abuse. We thank Jennifer Hobin for her assistance in running the experiment. Address correspondence to H. Rachlin, Department of Psychology, State University of New York, Stony Brook, NY 11794-2500 (e-mail: howard.rachlin@ sunysb.edu).
1984; Rapoport \& Chammah, 1965): When Player A cooperates, Player B, playing tit-for-tat, will cooperate in turn; when Player A defects, Player B will defect in turn. In the example shown in Figure 1, repeated cooperation with an opponent playing tit-for-tat would yield greater reward ( 5 units per trial) than would repeated defection ( 2 units per trial). Alternation between cooperation and defection yields an average of 3.5 units per trial $[(1+6) / 2=3.5]$, better than repeated defection, but still worse than repeated cooperation.

Playing a prisoner's dilemma game against an opponent playing tit-for-tat is analogous to a test of self-control. In typical studies of self-control with nonhumans, the alternatives on a given trial are smaller-sooner versus largerlater reinforcers. Rachlin (2000) calls this "simple ambivalence." However, when a prisoner's dilemma game is played against an opponent playing tit-for-tat, the alternatives on a given trial are a smaller versus a larger reinforcer, both immediate; choice of the smaller reinforcer leads to larger overall reward; choice of the larger reinforcer leads to smaller overall reward. In other words, the immediate consequence of defection is an increase in reinforcement rate, but its delayed consequence is a decrease in overall reinforcement rate. Rachlin (2000) calls this "complex ambivalence." Complex ambivalence may be seen as a laboratory model of harmful addictions, where choice of an immediately larger reinforcer (the addictive activity) leads to lower overall reward.

Although humans can learn to cooperate (and thus show self-control) in a prisoner's dilemma game against tit-fortat (Baker \& Rachlin, 2001), it has been difficult to replicate this finding with nonhumans in the laboratory. To ensure that payoffs resemble a prisoner's dilemma, some laboratory studies have had pairs of nonhuman subjects, such as rats (Flood, Lendenmann, \& Rapoport, 1983; Gardner, Corbin, Beltramo, \& Nickell, 1984), blue jays (Clem- 


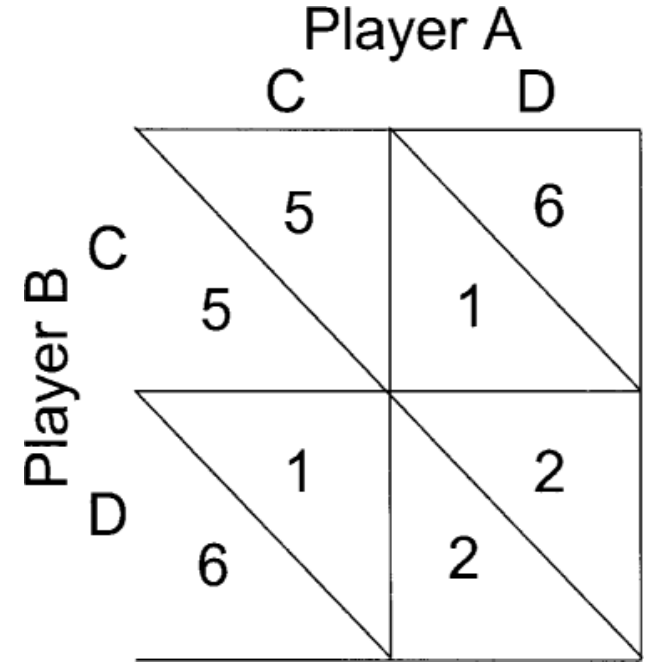

Figure 1. Contingency matrix in a typical two-player prisoner's dilemma game. The numbers in the matrix represent amounts of reward for two players (A and B) contingent on each player's choice between two alternatives: cooperate $(C)$ or defect $(D)$. If both players cooperate, each gets 5 points; if both defect, each gets 2 points. If one player cooperates while the other defects, the cooperator gets 1 point, whereas the defector gets 6 points.

ents \& Stephens, 1995), and starlings (Experiment 1 in Reboreda \& Kacelnik, 1993), simultaneously choose among food rewards contingent on the responses of both subjects. The subjects in all of these studies failed to cooperate when the contingencies resembled a prisoner's dilemma payoff matrix. On the basis of the results of these studies, however, it cannot be concluded that nonhumans are unable to learn cooperation. Even though these laboratory studies provided appropriate payoff contingencies, they did not control probability of reciprocation, because the other animal's choice was free to vary. ${ }^{1}$ Cooperation reflects self-control in the prisoner's dilemma only when the probability of reciprocation is sufficiently high. These studies provided an appropriate payoff matrix, but perhaps owing to a low probability of reciprocation by the nonhuman opponent, they may not have provided an incentive for the subjects to cooperate.

Green, Price, and Hamburger (1995) exposed individual pigeons to payoff matrices that appropriately modeled a dilemma, and they programmed the computer to use a titfor-tat contingency (1.0 probability of reciprocation). For example, in one of their conditions, pigeons chose between two keys, one representing cooperation and the other defection; the computer served as the "other player," reciprocating a pigeon's choice on the previous trial. On any given trial, a pigeon earned more food by defecting (one or three pellets) than by cooperating (zero or two pellets). But, over the long term, repeated cooperation earned two pellets per trial, whereas repeated defection earned one pellet per trial. Even though the probability of reciprocation was high (1.0), the pigeons generally defected. Why did the pigeons fail to cooperate (i.e., fail to show self-control)?
To obtain the higher reward contingent on cooperation in prisoner's dilemma games, behavior must be sensitive to the intertrial contingencies. Each trial in Green et al.'s (1995) study was $25 \mathrm{sec}$ in duration, which means that the advantage of cooperation could be obtained only if behavior and reinforcement were integrated across at least a 50 -sec time frame - well beyond that usually effective with pigeons. When salient stimuli are reliably paired with reinforcement, however, nonhumans can bridge even the long delays and can abstract relations between behavior and reinforcement characteristic of complex ambivalence (Heyman \& Tanz, 1995). One goal of the present study was to examine whether self-control (by pigeons playing against tit-for-tat) would be obtained by providing such salient and reliable stimuli.

Another goal of this study was to test whether temporal spacing of choices would affect level of cooperation. Delay between choice and reward presentation was held constant ( $3 \mathrm{sec})$, but the duration of the intertrial interval (ITI) was either 0 or $18 \mathrm{sec}$. If the prisoner's dilemma against a titfor-tat opponent is a self-control procedure, pigeons will be more likely to cooperate when the ITI (hence, delay of reinforcement) is short than when it is long (Mazur, 1987; Rachlin \& Green, 1972).

Several studies have suggested that sensitivity to overall reinforcement rate is heightened when choices are not evenly spaced but presented in fixed temporal patterns (Kudadjie-Gyamfi \& Rachlin, 1996; Rachlin \& Siegel, 1994; Siegel \& Rachlin, 1995; Silverstein, Cross, Brown, \& Rachlin, 1998; summarized in Rachlin, 1995). Silverstein et al. found that grouping several choices close together, followed by a long delay and then another group of choices, and so forth increased level of cooperation by human subjects playing a prisoner's dilemma game. A third goal of this study, therefore, was to test whether presenting choices in a similar fashion would increase the level of cooperation by pigeons.

\section{METHOD}

\section{Subjects}

Six male White Carneau pigeons, with previous experience pecking response keys for access to mixed grain in operant chambers, were maintained at $80 \%$ of their caged free-feeding weights by supplementary feeding, if necessary, following experimental sessions. The pigeons were housed individually in a colony room where they were provided continuous access to grit and vitamin-enriched water.

\section{Apparatus}

Experimental sessions were conducted, individually, in standard Coulbourn Instruments modular test chambers, approximately $25 \mathrm{~cm}$ wide, $28 \mathrm{~cm}$ long, and $30 \mathrm{~cm}$ high. The chambers were enclosed in sound- and light-attenuating boxes equipped with a ventilating fan. In the chamber, there was a houselight located at the top of center of the front panel, with its light deflected upward. The front panel also contained two response keys; each was $2.5 \mathrm{~cm}$ in diameter, with their centers located $6.5 \mathrm{~cm}$ from the ceiling. The distance between the two keys was $17 \mathrm{~cm}$, and they could be illuminated from behind by colored lights.

Effective keypecks required a force of approximately $0.2 \mathrm{~N}$ and produced a feedback click. Food reinforcement was access to mixed 


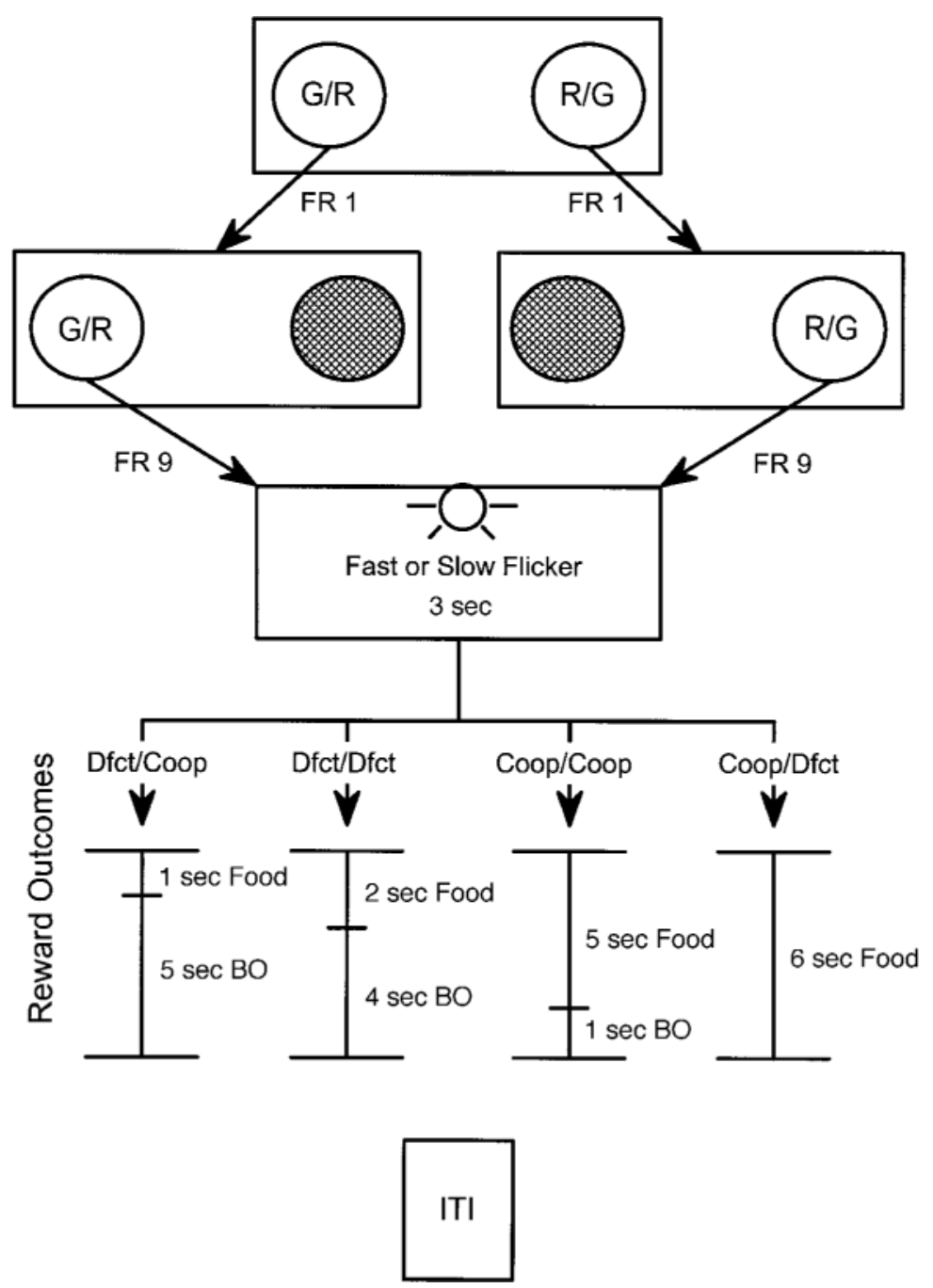

Figure 2. The sequence of events that occurred during a trial in all of the conditions except the defection condition in Phase 3, depending on a subject's choice in the previous and current trials. During the defection condition in Phase 3, the houselight always flickered slowly, and the subjects received only the two leftmost reward outcomes.

grain delivered from a hopper located in the center of the front panel, $2.54 \mathrm{~cm}$ from the grid floor. During reinforcement, the hopper was illuminated with white light, and the houselight and keylights were extinguished. A computer located in an adjoining room arranged experimental events and recorded data, using L2T2 software.

\section{Procedure}

Figure 2 shows the sequence of events during all the trials in all phases and conditions. A trial began with the two keys illuminated, one green and one red. The green key was always the cooperation choice; the red key was always the defection choice. The side on which the cooperation key appeared varied according to the phase of the experiment. The first peck on either key caused the other keylight to extinguish. The light on the chosen key extinguished after another nine pecks on that key, and then the houselight on the front panel flickered on and off either quickly (on for $100 \mathrm{msec}$, off for $100 \mathrm{msec}$ ) or slowly (on for $500 \mathrm{msec}$, off for $500 \mathrm{msec}$ ) for $3 \mathrm{sec}$. The flicker rate signaled both previous and subsequent events: whether the pigeon cooperated or defected on the previous trial (quickly if the pigeon cooperated and slowly if it defected); hence, the flicker rate also signaled whether the subject would obtain a relatively large reward or a relatively small reward (quickly before a large reward, slowly before a small reward) on the present trial.

Relatively large rewards consisted of 5- or 6-sec access to food, whereas relatively small rewards consisted of only 1- or 2-sec access to food. After flickering for $3 \mathrm{sec}$, the houselight was extinguished, and one of the four possible rewards was presented. The payoff contingencies corresponded to Player A's payoff matrix of Figure 1 with Player B playing tit-for-tat. If a pigeon defected on the previous trial and then cooperated on the current trial (Dfct/Coop), it obtained 1sec access to mixed grain, followed by a 5-sec blackout, during which the chamber was dark. If a pigeon defected on both previous and current trials (Dfct/Dfct), it obtained 2-sec access to mixed grain, followed by a 4-sec blackout. If a pigeon cooperated on both previous and current trials (Coop/Coop), it obtained 5-sec access to mixed grain, followed by a 1-sec blackout. Lastly, if a pigeon cooperated on 
Table 1

Experimental Conditions and Number of Sessions for Each Subject

\begin{tabular}{|c|c|c|c|c|c|c|c|c|}
\hline \multirow[b]{2}{*}{ Phase } & \multirow{2}{*}{$\begin{array}{c}\text { Duration of } \\
\text { Intertrial Interval } \\
\text { (ITI) in Seconds }\end{array}$} & \multirow{2}{*}{$\begin{array}{c}\text { Cooperation } \\
\text { Key Side } \\
\text { During } \\
\text { Session }\end{array}$} & \multicolumn{6}{|c|}{ Subject } \\
\hline & & & 1 & 2 & 3 & 4 & 5 & 6 \\
\hline 1 & 9 & either side & 12 & 12 & 12 & 12 & 12 & 12 \\
\hline 1 & 9 & left side & 7 & 7 & 7 & 7 & 7 & 7 \\
\hline 1 & 9 & right side & 7 & 7 & 7 & 7 & 7 & 7 \\
\hline 1 & 9 & either side & 12 & 12 & 12 & 12 & 12 & 12 \\
\hline 2 & 0 & either side & - & - & 25 & 24 & 24 & - \\
\hline 2 & 18 & either side & 31 & 24 & 27 & 27 & 33 & 24 \\
\hline 2 & 0 & either side & 24 & 24 & 27 & 36 & 30 & 27 \\
\hline 2 & 18 & either side & 40 & 39 & - & - & - & 36 \\
\hline 2 & Pattern & either side & 30 & 39 & 39 & 39 & 39 & 39 \\
\hline 3 & Pattern & alternate & 19 & 19 & 19 & 19 & 19 & 19 \\
\hline 3 & 18 & alternate & 24 & 24 & 24 & 24 & 24 & 24 \\
\hline 3 & 0 & alternate & 26 & 26 & 26 & 26 & 26 & 26 \\
\hline 3 & 0 (defect) & alternate & 46 & 47 & 46 & 47 & 47 & 47 \\
\hline
\end{tabular}

Note-The experimental conditions were presented in the order listed. During the Pattern conditions, all of the trials were followed by 0 -sec ITIs except every third trial, which was followed by an 18 -sec ITI. During Phase 3, the cooperation key switched sides between sessions. During the defection condition, the computer no longer played tit-for-tat; instead, it always defected.

the previous trial and then defected on the current trial (Coop/Dfct), it obtained a 6-sec access to mixed grain. After one of the four outcomes was presented, an ITI was initiated. The initiation of the ITI after a blackout was not signaled. The chamber remained dark during the ITI, just as it had been during the blackout period. Each session ended after 30 trials.

Phase 1. The purpose of Phase 1 was to assess side biases. During this phase, the duration of the ITI was always $9 \mathrm{sec}$. The side on which the cooperation key was presented during a session varied across three conditions (either side, left side, and right side). Table 1 shows the conditions, the number of sessions in each condition, and the order in which the conditions were presented to each subject. During the either-side conditions, the cooperation key (always green) was randomly ( $p=.5$, no other constraints) presented on either the left or the right side at the beginning of each trial. During the left-side condition, the cooperation key was presented only on the left side; during the right-side condition, it was presented only on the right side.

Phase 2. During this phase, the cooperation key was randomly presented on either side, and the ITI was varied across three conditions (0-sec, 18-sec, and pattern ITIs). During the pattern ITI condition, the subjects repeatedly received groups of three rapid trials separated by an 18-sec ITI. Each condition was in effect for a minimum of 24 sessions. Conditions were terminated once a subject's preference for cooperation appeared to be stable, based on visual inspection of the data.

Phase 3. During Phase 3, the cooperation key was presented on only one side during a session but alternated sides between sessions. This was done to ensure that the results of Phase 2 reflected preference for cooperation, rather than a side bias. During Phase 2, the subjects could alternate between cooperation and defection by simply responding on one side, because the cooperation key side was randomly assigned. In Phase 2 , the subjects, by responding on one side, would have received an average of 3.5-sec access to food per trial-more than the reward for repeatedly defecting (2-sec access to food), but less than that for repeatedly cooperating (5-sec access to food). Thus, in Phase 2, level of cooperation could reflect a side bias or a preference for alternating between cooperation and defection. To choose a mixture of cooperation and defection responses during Phase 3, the pigeons would have to peck on both keys. Furthermore, a side bias would result in drastic changes in overall reinforcement rate from one session to the next. The duration of the ITI was varied as in Phase 2. During the last condition (defection), the ITI duration was $0 \mathrm{sec}$, but the subjects were no longer exposed to tit-for-tat contingencies. Instead, the computer always defected. In other words, the houselight always flickered slowly after a choice response, and the subjects could receive only one of the two leftmost reward outcomes in Figure 2. During this condition, defection maximized reinforcement in both the short term and the long term, because the computer no longer reciprocated cooperation. The subjects were exposed to this condition to determine whether they still were sensitive to payoff contingencies at the end of the experiment. As in Phase 2, there was a minimum of 24 sessions of each condition, and the conditions were terminated once preference for cooperation appeared stable (based on visual inspection).

\section{RESULTS}

\section{Phase 1}

Pigeons 4, 5, and 6 exhibited a right key side bias (a low level of cooperation when the cooperation key was always on the left side, a high level of cooperation when the cooperation key was on the right side, and a percentage of cooperation choices of around 50\% during the either-side condition). The side bias of Pigeon 5 diminished during the second exposure to the either-side condition, in which it cooperated at a level of about $70 \%$, whereas side biases continued with Pigeons 4 and 6 . Of the 3 subjects that did not show a side bias, Pigeons 1 and 2 preferred cooperation, whereas Pigeon 3 preferred defection.

\section{Phases 2 and 3}

Figure 3 shows the median percentage of cooperation choices during the last five sessions in each condition during Phases 2 and 3. During Phase 2, Pigeons 1, 2, and 6 received the 18-sec ITI condition twice, and Pigeons 3, 4, and 5 received the 0 -sec ITI condition twice. Two dependent $t$ tests did not reveal a significant difference between the median percentages of cooperation choices during the first and the second exposures to the 0 - and 18 -sec ITI 
Subject \#1
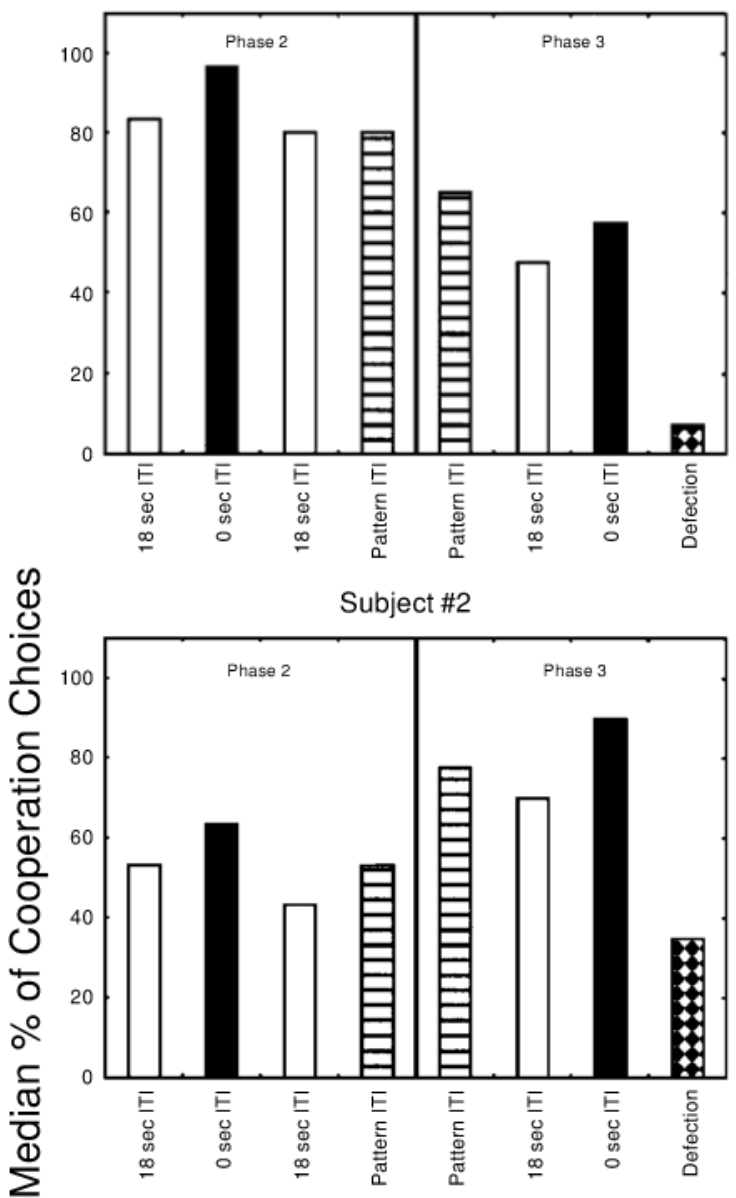

Subject \#3

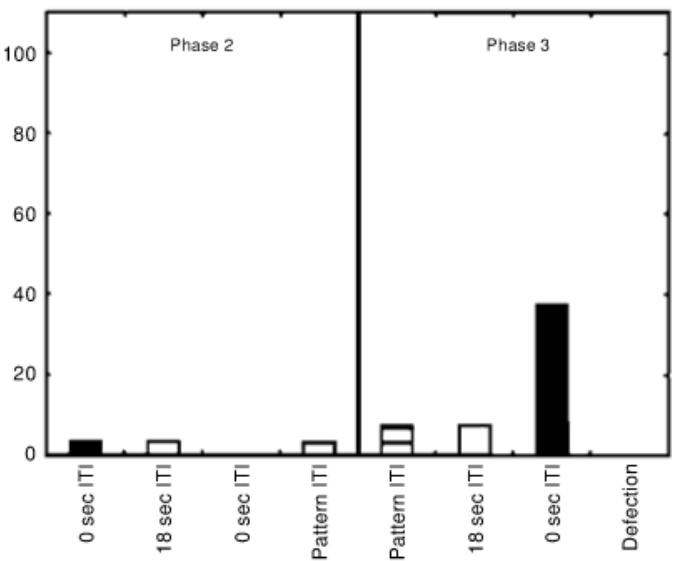

Subject \#4

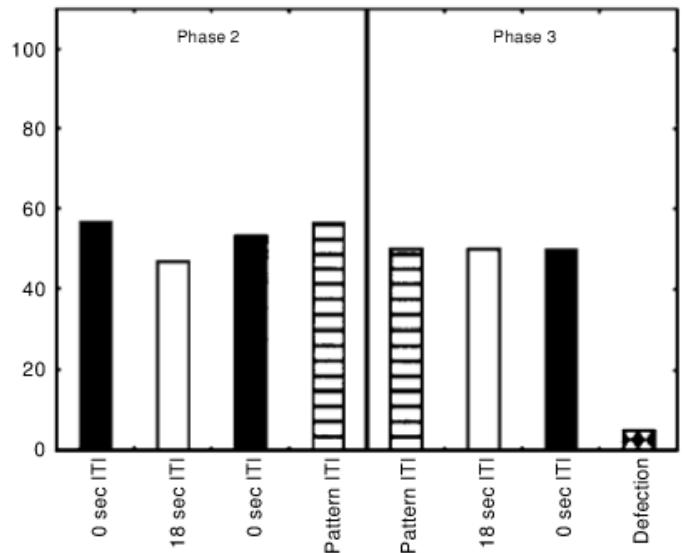

Subject \#5

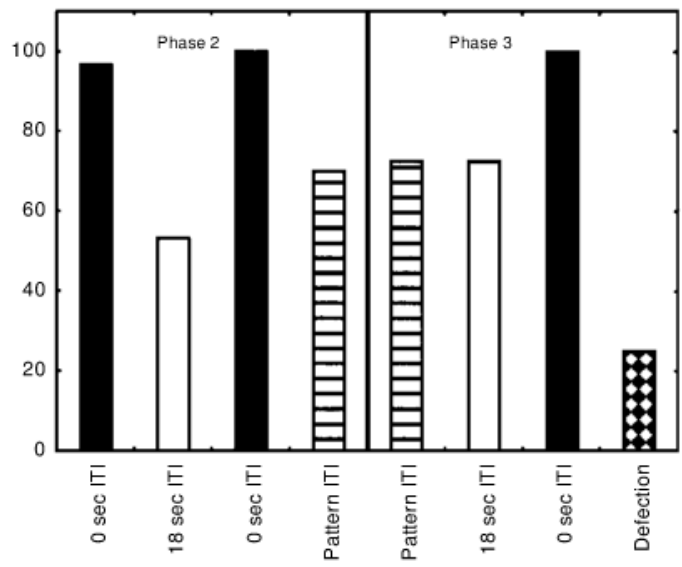

Subject \#6

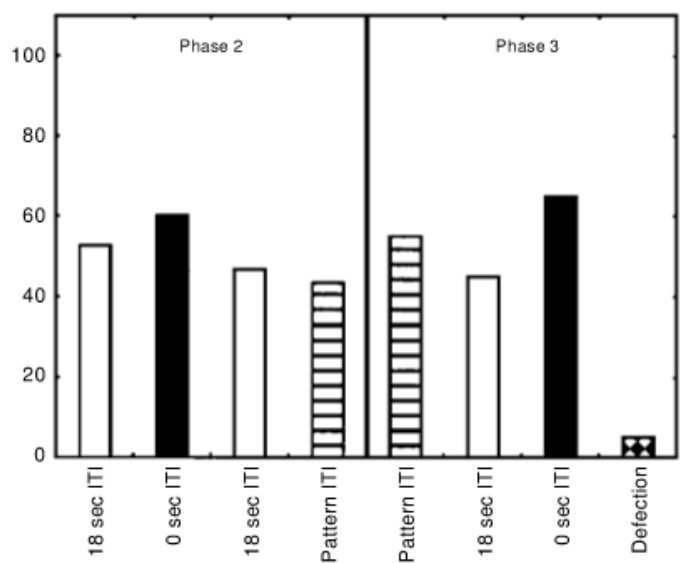

Condition

Figure 3. Median percentage of cooperation choices during the last five sessions in each condition during Phases 2 and 3 for each subject.

conditions $[t(2)=0.5, p=.67$, and $t(2)=2.5, p=.13$, respectively]. Therefore, one score for level of cooperation during the 0- and 18-sec ITI conditions in Phase 2 was computed for each subject by averaging the median per- centage of cooperation choices across the two exposures to the same condition.

Three dependent $t$ tests compared median percentages of cooperation choices during the $0-\mathrm{sec}, 18$-sec, and pat- 
tern ITI conditions in Phase 2 with the same conditions in Phase 3. Level of cooperation did not differ between phases $[t(5)=0.18, p=.87, t(5)=0.22, p=.84$, and $t(5)=$ $0.62, p=.56$, respectively]. Therefore, one level of cooperation was computed for each of the three ITI conditions ( 0 -sec, 18 -sec, and pattern ITI) for each subject by averaging median percentages of cooperation choices across all of the respective conditions in both Phase 2 and Phase 3.

A repeated measures analysis of variance showed a significant difference in level of cooperation during the three ITI conditions $[F(2,10)=9.24, p<.01]$. A post hoc Scheffé test revealed that level of cooperation was significantly higher during the 0 -sec ITI condition $(M=.64)$ than during both the $18-\sec (M=.48)$ and the pattern $(M=$ $.53)$ ITI conditions ( $p<.01$ and $p=.04$, respectively). However, there was no significant difference in level of cooperation during the 18 -sec and pattern ITI conditions $(p=.49)$.

In a dependent measures $t$ test, the median percentage of cooperation choices during the defection condition was compared with the level of cooperation (as computed for the previous analysis) during the 18 -sec ITI condition. Level of cooperation was significantly lower during the defection condition $(M=.13)$ than during the 18 -sec ITI condition $[M=.48 ; t(5)=4.35, p<.01]$.

\section{DISCUSSION}

The results of this study demonstrate that when their choices are reciprocated, pigeons can learn to cooperate (and thus show self-control) during a repeated prisoner's dilemma game. Moreover, the temporal spacing of choices affected level of cooperation. When the probability of reciprocation was 1.0 (making cooperation optimal in the long term), half of the subjects cooperated rather than defected. On the other hand, when the computer stopped reciprocating cooperation (defection condition), all of the subjects defected. Level of cooperation also fluctuated as a function of the delay between trials. That is, when cooperation and defection were reciprocated, level of cooperation was higher when trials were presented close together than when they were temporally separated by an 18 -sec ITI.

The patterning of choices used in this experiment did not significantly increase level of cooperation, as compared with the condition in which all of the trials were separated by 18 -sec ITIs. The effectiveness of patterning in increasing cooperation depends on its enhancement of integration of contingencies across trials (Rachlin, 2000). It is possible that, in this experiment, the signals overshadowed the patterning effect for those subjects that learned to cooperate. Perhaps some other patterning procedure (for example, two rapid trials separated by a longer interval) might have succeeded.

Two factors may explain why some of the pigeons in the present study cooperated, whereas those in Green et al.'s (1995) study did not. Both studies used prisoner's dilemma payoff matrices and programmed the computer to recip- rocate choices according to a tit-for-tat strategy. One difference between the studies is that the trials in Green et al.'s study were always $25 \mathrm{sec}$ in duration; as the present study demonstrates, this relatively long interval may have contributed to the low level of cooperation. Another crucial difference may have been the way the relation between a choice and the magnitude of a reward was signaled.

Heyman and Tanz (1995) found that pigeons' choice behavior was more sensitive to overall rates of reinforcement when deviations from it were signaled than when they were unsignaled. They suggested that the mechanism behind their findings was a strengthening of certain response classes congruent with maximizing overall reinforcement rate. The stimulus conditions in the present study may have strengthened a response class consisting of repeated cooperation, whereas the stimulus conditions in Green et al.'s (1995) study did not. In the present study, a response class of repeated cooperation may have been strengthened in the following manner. After 10 pecks on a key, the key was darkened, and there was a 3-sec delay to reward. During this delay, the houselight flickered on and off. The frequency with which the houselight flickered (quickly or slowly) was perfectly correlated with two events: (1) choice on the previous trial and (2) whether the subject was about to receive one of the two relatively large rewards (5- or 6-sec access to food) or one of the two relatively small rewards (1- or 2-sec access to food). Therefore, across trials, there was a strong correlation between cooperation and flicker rate (and, in turn, between flicker rate and reinforcement magnitude). The results show that these relations gained control over the behavior of some of the pigeons. Thus, the houselight strengthened a response class (cooperation) congruent with maximizing overall reinforcement rate.

The stimulus conditions in Green et al.'s (1995) study differed from those of the present study in that, after subjects made a choice, the key they chose remained lit for $6 \mathrm{sec}$. During the final $3 \mathrm{sec}$, either a blue or a yellow cuelight was illuminated, depending on a subject's choice in the previous trial (just like the flicker rate of the houselight in the present experiment). The stimuli presented between choice and outcome in the Green et al. study thus differed in two ways from those of the present study. First, in the Green et al. study, one of four stimulus combinations (signaling the four previous-response and presentresponse combinations) was presented before each reward, whereas in the present study, only one of two stimuli (signaling only the previous response) was presented. Thus, the discrimination to be learned in the present study was simpler. Second, in the present study, the absence of a signal correlated with the present-trial response (the choice just made) may have caused the subjects to be less sensitive to present-trial contingencies and more sensitive to the (signaled) reward magnitudes contingent on their previous choice (5 and 6 vs. 1 and 2).

Sensitivity to present-trial and intertrial contingencies may have also differed between Green et al.'s (1995) study and the present study, since they used different payoff values. In Green et al.'s study, subjects had a choice between 
2 and $0 \mathrm{sec}$ of food when the computer cooperated and a choice between 3 and $1 \mathrm{sec}$ of food when the computer defected, whereas the subjects in the present study had a choice between 6 and $5 \mathrm{sec}$ on cooperation trials and a choice between 2 and $1 \mathrm{sec}$ on defection trials. Thus, the subjects in Green et al.'s study received, on average, only a $1-\mathrm{sec}$ increase in access to food on the next trial when they moved from a defection trial to a cooperation trial, whereas the subjects in the present study received, on average, a 4-sec increase. Furthermore, the subjects in Green et al.'s study experienced, on average, a 2-sec increase in access to food on the current trial by defecting, whereas the subjects in the present study received, on average, only a 1 -sec increase. Thus, in the present study, it may have been easier to distinguish the changes in reward magnitudes that were due to intertrial contingencies than to distinguish the changes in reward magnitudes that were due to present-trial contingencies. However, even under the present conditions, not all of the pigeons learned to cooperate. There is clearly room for improvement.

In conclusion, the present study demonstrates that nonhuman animals can learn to cooperate and, thus, show self-control during the prisoner's dilemma game even though cooperating reduces reinforcement in the short term. However, level of cooperation decreased as temporal spacing of choices increased, which suggests that solving dilemmas and problems of self-control may hinge on the salience of temporally extended events.

\section{REFERENCES}

Axelrod, R. (1984). The evolution of cooperation. New York: Basic Books.

BAKER, F., \& RACHLIN, H. (2001). Probability of reciprocation in repeated prisoner's dilemma games. Journal of Behavioral Decision Making, 14, 51-67.

Clements, K. C., \& Stephens, D. W. (1995). Testing models of non-kin cooperation: Mutualism and the prisoner's dilemma. Animal Behaviour, 50, 527-535.

Flood, M., Lendenmann, K., \& Rapoport, A. (1983). $2 \times 2$ games played by rats: Different delays of reinforcement as payoffs. Behavioral Science, 28, 65-68.

Gardner, R. M., Corbin, T. L., Beltramo,J. S., \& Nickell, G. S. (1984).
The prisoner's dilemma game and cooperation in the rat. Psychological Reports, 55, 687-696.

Green, L., Price, P. C., \& Hamburger, M. E. (1995). Prisoner's dilemma and the pigeon: Control by immediate consequences. Journal of the Experimental Analysis of Behavior, 64, 1-17.

Heyman, G. M., \& TANZ, L. (1995). How to teach a pigeon to maximize overall reinforcement rate. Journal of the Experimental Analysis of Behavior, 64, 277-297.

Kudadje-Gyamfi, E., \& Rachlin, H. (1996). Temporal patterning in choice among delayed outcomes. Organizational Behavior \& Human Decision Processes, 65, 61-67.

Mazur, J. E. (1987). An adjusting procedure for studying delayed reinforcement. In M. L. Commons, J. G. Mazur, J. A. Nevin, \& H. Rachlin (Eds.), Quantitative analyses of behavior: Vol. 5. Reinforcer value: The effect of delay and intervening events on reinforcement value (pp. 5573). Hillsdale, NJ: Erlbaum.

RACHLIN, H. (1995). The value of temporal patterns in behavior. Current Directions in Psychological Science, 4, 188-191.

RACHLIN,H. (2000). The science of self-control. Cambridge, MA: Harvard University Press.

RACHLIN,H., \& GREEN, L. (1972). Commitment, choice, and self-control. Journal of the Experimental Analysis of Behavior, 17, 15-22.

RACHLIN, H., \& SiEgEL, E. (1994). Temporal patterning in probabilistic choice. Organizational Behavior \& Human Decision Processes, 59, 161-176.

Rapoport, A., \& Chammah, A. (1965). Prisoner's dilemma. Ann Arbor: University of Michigan Press.

Reboreda, J. C., \& KaCELnIK, A. (1993). The role of autoshaping in cooperative two-player games between starlings. Journal of the Experimental Analysis of Behavior, 60, 67-83.

SiEgel,E, \& RACHLIN,H. (1995). Soft commitment: Self-control achieved by response persistence. Journal of the Experimental Analysis of Behavior, 64, 117-128.

Silverstein, A. Cross, D., Brown, J., \& Rachlin, H. (1998). Prior experience and patterning in a prisoner's dilemma game. Journal of Behavioral Decision Making, 11, 123-138.

\section{NOTES}

1. Reboreda and Kacelnik (1993) controlled probability of reciprocation in their Experiments 3 and 4 and found higher levels of cooperation. It is difficult to interpret their results, however, because their subjects cooperated by responding on a key and defected by withholding a response. Therefore, as the authors suggested, level of cooperation was confounded with an autoshaping process.

(Manuscript received December 30, 2000; revision accepted for publication October 2, 2001.) 